\title{
New Results of Measurements in the Neutrino-4 Experiment
}

\author{
A. P. Serebrov and R. M. Samoilov \\ NRC “KI" Petersburg Nuclear Physics Institute, Gatchina 188300, Russia
}

\begin{abstract}
We present new results of measurements of reactor antineutrino flux and spectrum dependence on the distance in the range 6-12 meters from the center of the reactor core at SM-3 reactor (Dimitrovgrad, Russia). Additional measurements were carried out and set of data to perform statistical analysis was almost doubled since the previous report. Using all collected data, we performed the model independent analysis on the oscillation parameters $\Delta m_{14}^{2}$ and $\sin ^{2} 2 \theta_{14}$. The method of coherent summation of results of measurements allows us to directly observe the effect of oscillations. We observed an oscillation effect at CL 3.0 $\sigma$ in vicinity of $\Delta m_{14}^{2} \approx 7.25 \mathrm{eV}^{2}$ and $\sin ^{2} 2 \theta_{14} \approx 0.26 \pm 0.08(3.0 \sigma)$. We provide a comparison of our results with results of other experiments on search for sterile neutrino. Combining the result of the Neutrino-4 experiment and the results of measurements of the gallium anomaly and reactor anomaly we obtained value $\sin ^{2} 2 \theta_{14} \approx 0.19 \pm 0.04(4.6 \sigma)$.
\end{abstract}

Keywords: neutrino oscillation, sterile neutrino

DOI: 10.31526/ACP.NDM-2020.26

\section{INTRODUCTION}

Experimental search for possible existence of neutrino oscillation into sterile state have been carried out for many years. That idea is under consideration in experiments carried out at reactors, artificial neutrino sources and others [1]-[10]. Sterile neutrino can be considered as a candidate for the dark matter.

The hypothesis of oscillation can be verified by direct measurement of the antineutrino flux and energy spectrum versus distance at short 6-12 m distances from the reactor core. We use the method of relative measurements, which can be more precise. This method requires a detector to be movable and spectrum sensitive. To detect oscillations to a sterile state, one needs to observe a deviation of flux-distance relation from $1 / L^{2}$ dependence and alteration of the form of energy spectrum with distance. If such process does occur, it can be described at short distances by the equation:

$$
P\left(\bar{v}_{e} \rightarrow \bar{v}_{e}\right)=1-\sin ^{2}\left(2 \theta_{14}\right) \sin ^{2}\left(\frac{\Delta m_{14}^{2}\left[\mathrm{eV}^{2}\right] L[\mathrm{~m}]}{E_{\bar{v}_{e}}[\mathrm{MeV}]}\right),
$$

where $E_{\bar{v}_{e}}$ is antineutrino energy in $\mathrm{MeV}, \mathrm{L}$ - distance in meters, $\Delta m_{14}^{2}$ is difference between squared masses of electron and sterile neutrinos, $\theta_{14}$ is mixing angle of electron and sterile neutrinos. For the experiment to be conducted, one needs to carry out measurements of the antineutrino flux and spectrum as near as possible to a practically point-like antineutrino source.

The detector scheme with active and passive shielding is shown in Figure 1. More detailed description of neutrino lab preparation, background measurements and full-scale detector measurements scheme are in $[7,8]$.

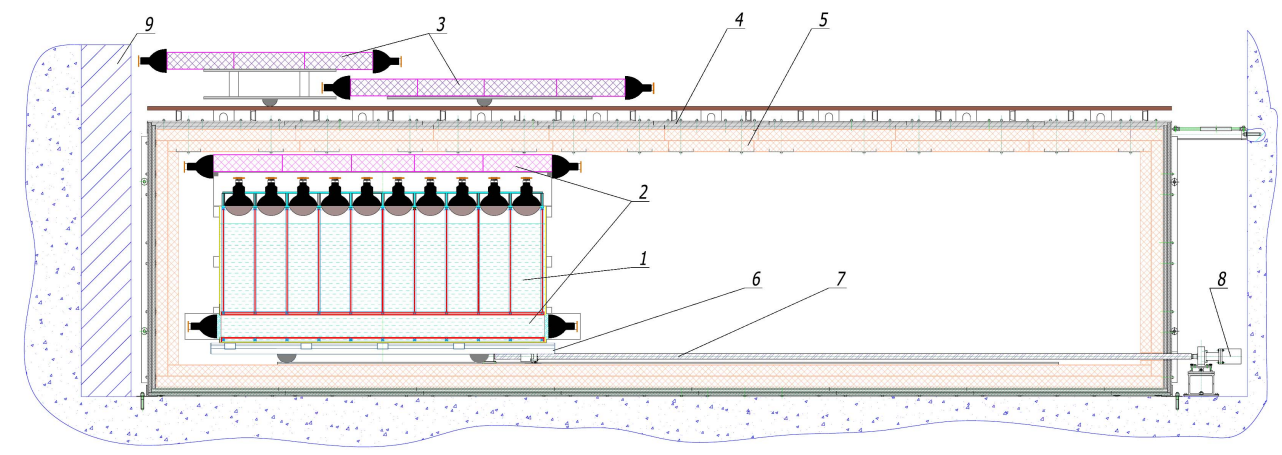

FIGURE 1: General scheme of an experimental setup. 1 - detector of reactor antineutrino, 2 - internal active shielding, 3 - external active shielding (umbrella), 4 - steel and lead passive shielding, 5 - borated polyethylene passive shielding, 6 - moveable platform, 7 - feed screw, 8 - step motor, 9 -shielding against fast neutrons made of iron shot. 


\section{THE MATRIX OF MEASUREMENTS}

The results of experimental measurements of the antineurino flux dependence on distance and energy of antineurino can be presented in the form of a matrix, which contains 216 elements, where $N_{i k}$ is difference of ON - OFF rates for i-th interval of energy and for k-th distance from reactor core. The energy spectrum is divided into 9 intervals of $500 \mathrm{keV}$, which corresponds to the energy resolution of the detector. The distance step corresponds to the cell size of $23 \mathrm{~cm}$. In total there are 24 positions of antineurino flux measurements from $6.4 \mathrm{~m}$ to $11.9 \mathrm{~m}$. Also more detailed data representations with division into energy intervals $125 \mathrm{keV}$ and 250 $\mathrm{keV}$ were used.

\section{SCHEME OF THE EXPERIMENTAL DATA ANALYSIS}

There is a well-known problem of discrepancy between the experimental and calculated spectra, which also manifests itself in our experiment [8]. Therefore, the method of the analysis of the experimental data should not rely on a precise knowledge of the energy spectrum. Therefore, we propose a model-independent method of data analysis, which employs equation (2), where the numerator is the rate of antineutrino events per $10^{5} \mathrm{~s}$ with a correction to geometric factor $L^{2}$ and denominator is the antineutrino events rate averaged over all distances:

$$
\begin{gathered}
\frac{\left(N_{i k} \pm \Delta N_{i k}\right) L_{k}^{2}}{K^{-1} \sum_{k}^{K}\left(N_{i k} \pm \Delta N_{i k}\right) L_{k}^{2}}= \\
\frac{1-\sin ^{2} 2 \theta_{14} \sin ^{2} \frac{1.27 \Delta m_{14}^{2} L_{k}}{E_{i}}}{K^{-1} \sum_{k}^{K}\left(1-\sin ^{2} 2 \theta_{14} \sin ^{2} \frac{1.27 \Delta m_{14}^{2} L_{k}}{E_{i}}\right)}
\end{gathered}
$$

Equation (2) can be used to do a model-independent analysis of data because the left part includes only experimental data $k=$ $1,2, \ldots, K$ for all distances in the range $6.4-11.9 \mathrm{~m}, K=24 ; i=1,2, \ldots, 9$ corresponding to $500 \mathrm{keV}$ energy intervals in range 1.5 $\mathrm{MeV}$ to $6.0 \mathrm{MeV}$. The right part is the same ratio obtained within the oscillation hypothesis. The left part is normalized to spectrum averaged over all distances; hence the oscillation effect is considerably averaged out in the denominator if oscillations are frequent enough in the considered range of distances.

\section{MONTE CARLO CALCULATION}

In this section we present results of MC simulation in which we incorporated geometric configuration of the antineutrino source and detector including the sectioning. In this simulation we have already used optimal parameters $\Delta m_{14}^{2}$ and $\sin ^{2} 2 \theta_{14}$, which were derived from the analysis of experimental data.

The source of antineutrino with geometrical dimensions of the reactor core $42 \times 42 \times 35 \mathrm{~cm}^{3}$ was simulated, as well as a detector of antineutrino considering its geometrical dimensions ( 50 sections of $22.5 \times 22.5 \times 85 \mathrm{~cm}^{3}$ ). The antineutrino spectrum of $\mathrm{U}^{235}$ increased by function of oscillations $1-\sin ^{2} 2 \theta_{14} \sin ^{2}\left(1.27 \Delta m_{14}^{2} L_{k} / E_{i}\right)$ was used. Though it did not matter which energy spectrum of antineutrino we use since it is reduced in the equation (2). The most important parameter in this simulation was the energy resolution of the detector, which was $500 \mathrm{keV}$. Fig. 2 (left) shows the relationship of the oscillation pattern to the energy resolution of the detector. The oscillation curve corresponding to experimental energy resolution of the detector has to give the best fit of experimental data. Fig. 2 (right) shows the simulated matrix of ratio $\left(N_{i k} \pm \Delta N_{i k}\right) L_{k}^{2} / K^{-1} \sum\left(N_{i k} \pm \Delta N_{i k}\right) L_{k}^{2}$ for calculations with $\Delta N_{i k} / N_{i k}$ equal to $1 \%$, which is significantly better than the experimental value. One also can see a picture of the process of oscillations on the plane $(E, L)$ and as dependence on parameter $L / E$.

The presented MC simulation reveals that resolution of the detector is extremely important for detecting the effect of the oscillations. Moreover, the oscillation effect could be extracted from data only by using the experimental dependence of ratio $\left(N_{i k} \pm \Delta N_{i k}\right) L_{k}^{2} / K^{-1} \sum\left(N_{i k} \pm \Delta N_{i k}\right) L_{k}^{2}$ on the parameter $L / E$. It should be noted that integration of the matrix over energy or distance significantly suppresses the ability to detect the effect of oscillations. Besides, the measurements in range $6-9 \mathrm{~m}$ are especially important, while measurements in range $9-12 \mathrm{~m}$ do not bring a significant contribution in sensitivity of the experiment, but they are used to correctly normalize the results of measurements.

\section{ANALYSIS OF THE EXPERIMENTAL RESULTS FOR OSCILATION SEARCH}

The matrix of measurements incorporates data about the dependance of antineutrino flux on distance and energy. The elements of the matrix $R_{i k}$ represents the difference ON-OFF signal in the i-th energy interval and k-th interval of distance to the center of the reactor core. This matrix should be compared with a calculated MC matrix, an example of such matrix is shown in Fig. 2 on the 

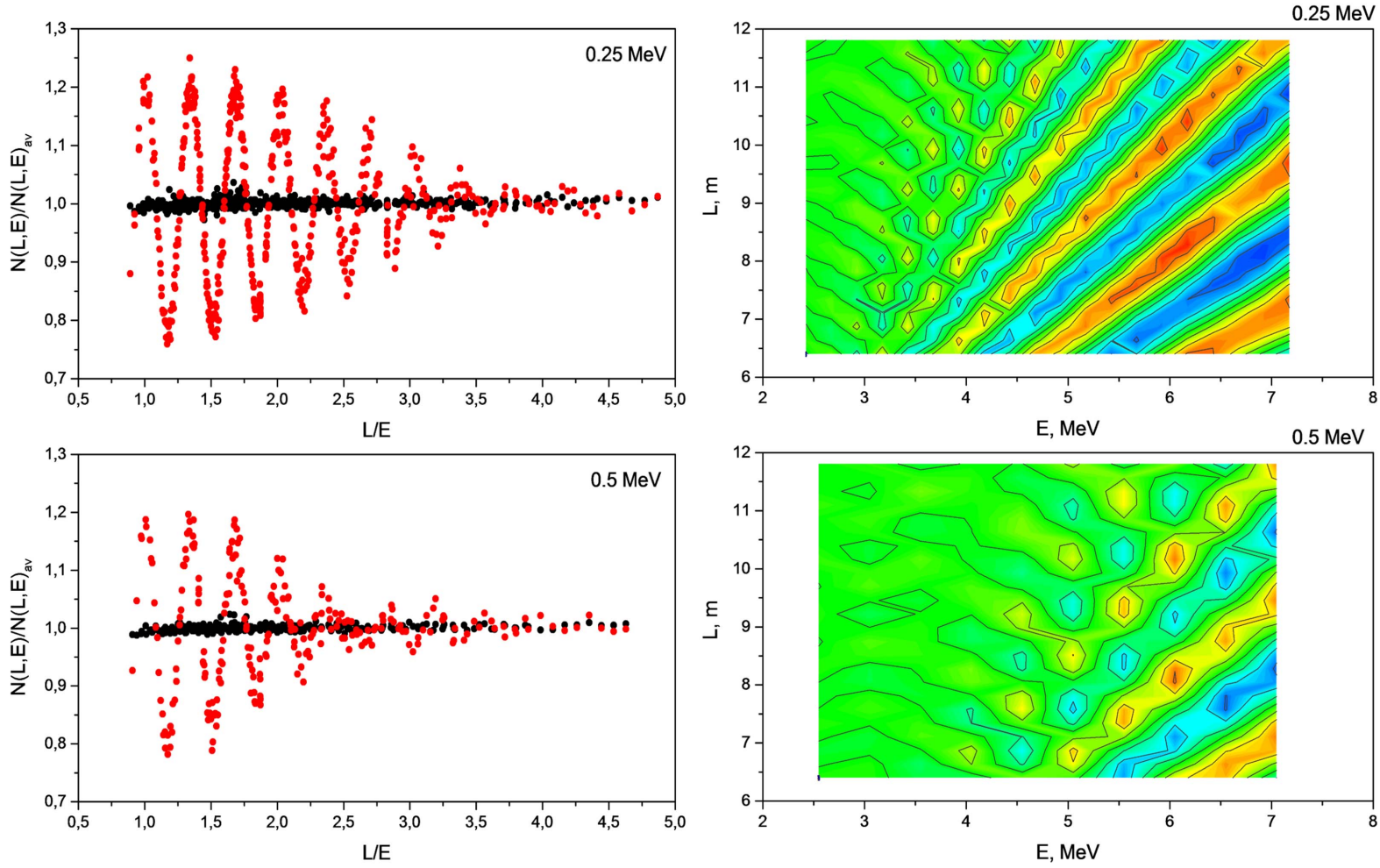

FIGURE 2: The simulated matrix of ratio $\left(N_{i k} \pm \Delta N_{i k}\right) L_{k}^{2} / K^{-1} \sum\left(N_{i k} \pm \Delta N_{i k}\right) L_{k}^{2}$ for various energy resolutions of detector.

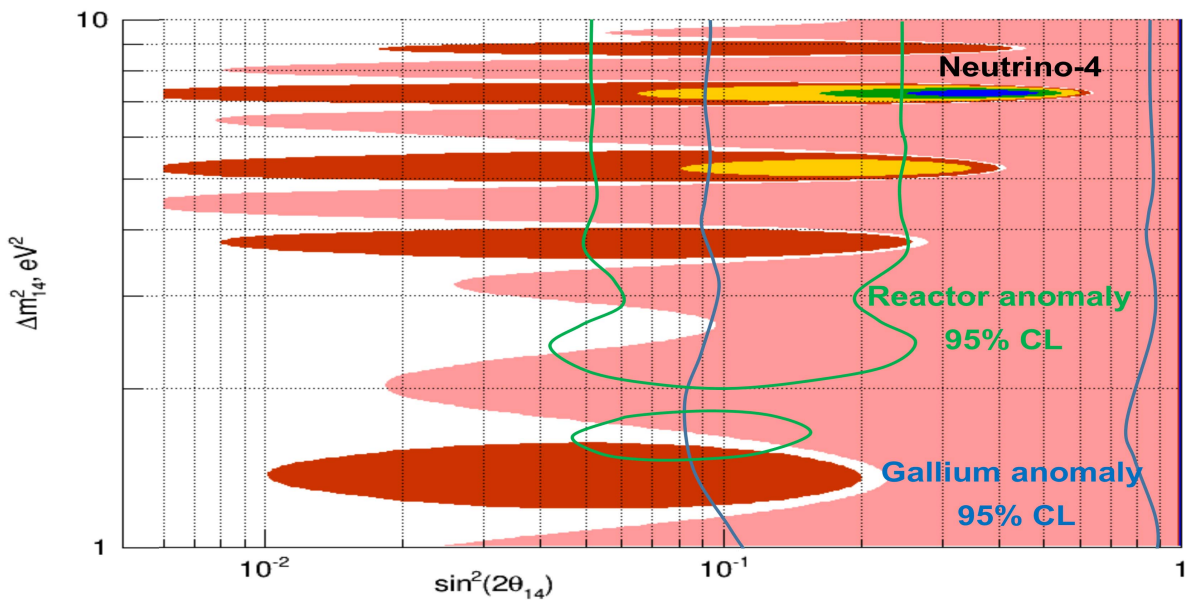

FIGURE 3: Restrictions on parameters of oscillation into sterile state with $99.95 \%$ CL (pink), area of acceptable with $99.73 \%$ CL values of the parameters (yellow), area of acceptable with $95.45 \%$ CL values of the parameters (green), area of acceptable with $68.30 \%$ CL values of the parameters (blue).

right.

$$
\begin{aligned}
& R_{i k}^{\exp }=\frac{\left(N\left(E_{i}, L_{k}\right) L_{k}^{2}\right)}{\left(K^{-1} \sum_{k}^{K} N\left(E_{i}, L_{k}\right) L_{k}^{2}\right.} \\
& =\frac{1-\sin ^{2} 2 \theta_{14} \sin ^{2}\left(\frac{1.27 \Delta m_{14}^{2} L_{k}}{E_{i}}\right)}{K^{-1} \sum_{k}^{K}\left(1-\sin ^{2} 2 \theta_{14} \sin ^{2}\left(\frac{1.27 \Delta m_{14}^{2} L_{k}}{E_{i}}\right)\right)}=R_{i k}^{\mathrm{th}}
\end{aligned}
$$

Comparison of experimental matrix with calculated MC matrix in (3) can be done using $\Delta \chi^{2}$ method.

$$
\sum_{i, k}\left(R_{i k}^{\exp }-R_{i k}^{\text {th }}\right)^{2} /\left(\Delta R_{i k}^{\exp }\right)^{2}=\chi^{2}\left(\sin ^{2} 2 \theta_{14}, \Delta m_{14}^{2}\right),
$$



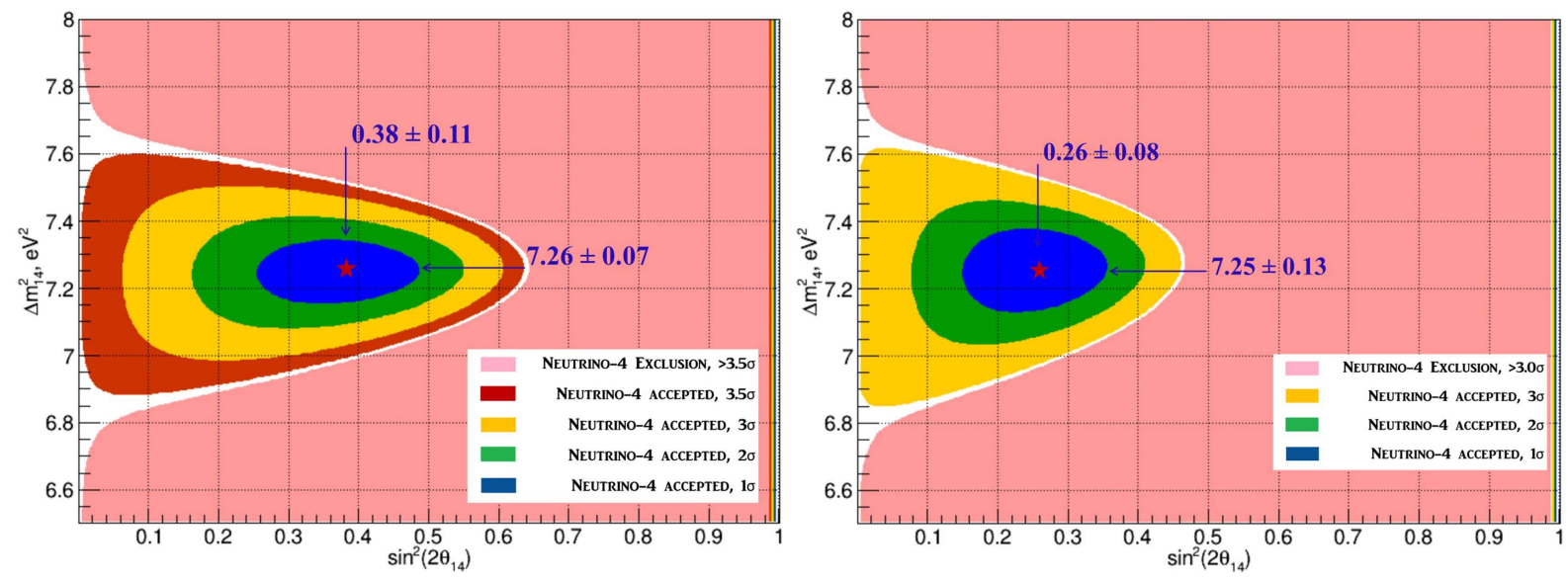

FIGURE 4: Left - Significantly magnified central area. Right - Confidence levels of the area around oscillation parameters obtained as the best fit in case of averaging over three data sets.

The result of the analysis of experimental data using $\Delta \chi^{2}$ method with equation (4) is shown in Fig. 3-4. The area of oscillation parameters colored in pink are excluded with CL more than $99.95 \%(>3.5 \sigma)$. However, in the area $\Delta m_{14}^{2}=(7.26 \pm 0.07) \mathrm{eV}^{2}$ and $\sin ^{2} 2 \theta_{14}=0.38 \pm 0.11$ the oscillation effect is observed at CL $99.73 \%(3 \sigma)$, and this area is followed by a few satellites. Minimal value of $\chi^{2}$ occurs at $\Delta m_{14}^{2} \approx 7.26 \mathrm{eV}^{2}$. Notice that uncertainty in determination of $\Delta m_{14}^{2}$ is closely related to the accuracy of energy calibration of the detector, which is estimated to be $10 \%$. However, in calculation of optimal value for $\Delta m_{14}^{2}$ we obtained several minimums of function $\Delta \chi^{2}\left(\Delta m_{14}^{2}, \sin ^{2} 2 \theta_{14}\right)$ located close to each other. That fact can result in variation of $\Delta m_{14}^{2}$ value giving the best fit after further data collection. Therefore, currently $\Delta m_{14}^{2}=(7.26 \pm 0.7) \mathrm{eV}^{2}$.

More detailed analysis of the experimental data was performed with division of the energy spectrum using various intervals: $125 \mathrm{keV}, 250 \mathrm{keV}$ and $500 \mathrm{keV}$. This analysis was aimed to avoid fluctuations in the final result caused by usage of some particular system of data division. For this purpose, we used 24 distance points (with $23 \mathrm{~cm}$ interval) and 9 energy intervals ( $0.5 \mathrm{MeV}$ bin) or $18(0.25 \mathrm{MeV})$ or $36(0.125 \mathrm{MeV})$.

Corresponding matrices included 216, 432 and 864 elements. To form dependence of ratio $\frac{\left(N_{i k} \pm \Delta N_{i k}\right) L_{k}^{2}}{K^{-1} \sum\left(N_{i k} \pm \Delta N_{i k}\right) L_{k}^{2}}$ on parameter $L / E$ we merged adjacent points into groups of 8,16 and 32 correspondingly. At the next step the obtained $L / E$ dependencies were averaged and consequently the fluctuations of data divisions were averaged out. The results of averaging of the data are shown in Figure 5 (black squares). In purpose of comparison, the results of analysis with interval $500 \mathrm{keV}$, which corresponds to energy resolution of the detector, are also presented (blue triangles).

In analysis with averaging over data sets with energy intervals $125 \mathrm{keV}, 250 \mathrm{keV}$ and $500 \mathrm{keV}$ (black squares in Fig. 5) the fit with the given above parameters has the goodness of fit $28 \%$, while fit with a constant equal to one (assumption of no oscillations) has the goodness of fit only $3 \%$. We obtained $\chi^{2} / D O F=20 / 17$ for the version with oscillation and $\chi^{2} / D O F=32 / 19$ for the version without oscillation. To achieve the stability of the results we chose the analysis with averaging of the data.

For reasons of reliability of the final result, we choose the case of data processing with averaging. Confidence levels of the area around oscillation parameters obtained as the best fit in case of averaging over three data sets is $3.0 \sigma-\Delta m_{14}^{2} \approx 7.25 \mathrm{eV}^{2}$ and $\sin ^{2} 2 \theta_{14} \approx 0.26 \pm 0.08$.

Study of possible systematic effects was performed using a background of fast neutrons created by cosmic rays. Fast neutrons mimics antineutrino registration signal because a recoil proton imitates signal of a positron. In order to study systematic effects, one has to turn off antineutrino flux (turn off the reactor) and perform the same analysis of collected data. The analysis resulted in disappearance of oscillation curve. Therefore, we confirmed that the apparatus does not produce systematical errors (more detail in [8])

In the Neutrino-4 experiment we measure the oscillation parameter $\sin ^{2} 2 \theta_{14}$, which is two times bigger than the deficiency of reactor antineutrino flux at large distance. In order to compare the results of Neutrino- 4 experiment with results of measurements of reactor and gallium anomalies the obtained value of parameter $\sin ^{2} 2 \theta_{14}$ can be turned into the flux deficiency and vice versa. We will compare results in terms of oscillation parameter $\sin ^{2} 2 \theta_{14}$.

The neutrino deficiency called gallium anomaly (GA) $[9,10]$ has oscillation parameter $\sin ^{2} 2 \theta_{14} \approx 0.32 \pm 0.10(3.2 \sigma)$. The result of reactor antineutrino anomaly (RAA) [11]-[13] measurements is $\sin ^{2} 2 \theta_{14} \approx 0.13 \pm 0.05(2.6 \sigma)$. Combination of these results gives an estimation for mixing angle $\sin ^{2} 2 \theta_{14} \approx 0.19 \pm 0.04(4.6 \sigma)$. 


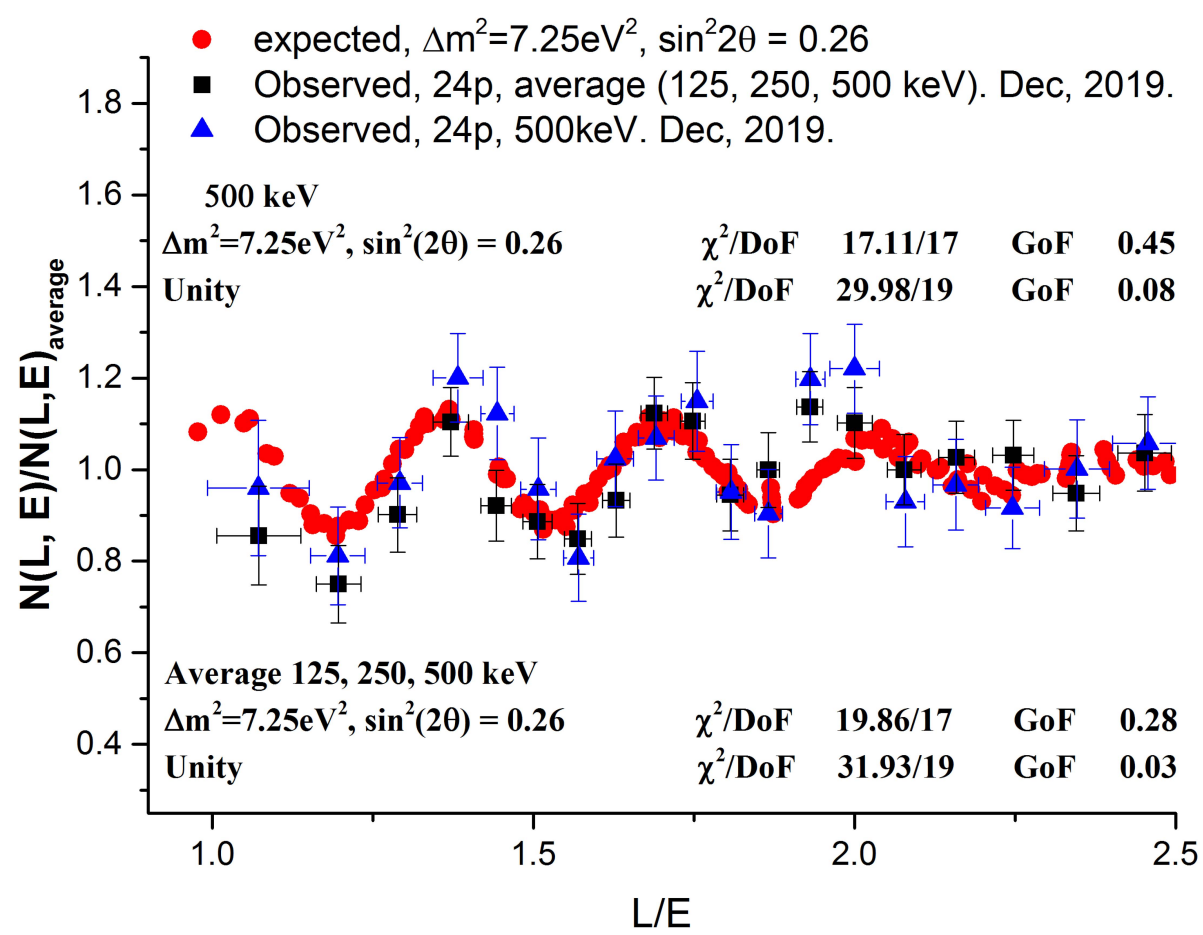

FIGURE 5: The results of data analysis with energy interval $500 \mathrm{keV}$, which corresponds to energy resolution of the detector (blue triangles). The results of data analysis with averaging over energy intervals $125 \mathrm{keV}, 250 \mathrm{keV}$ and $500 \mathrm{keV}$ (black squares).

\section{COMPARISON WITH KATRIN EXPERIMENT}

The values of oscillation parameters obtained in the Neutrino-4 experiment can be used to estimate mass of the electron antineutrino, using general formulas for neutrino model [14, 15] with extension to $3+1$ model:

$$
\begin{aligned}
m_{v_{e}}^{\text {eff }} & =\sqrt{\sum m_{i}^{2}\left|U_{e i}\right|^{2}}, \\
\sin ^{2} 2 \theta_{14} & =4\left|U_{14}\right|^{2}\left(1-\left|U_{14}\right|^{2}\right), \\
\Delta m_{14}^{2} & \approx m_{4}^{2}, \ldots,\left|U_{14}\right|^{2} \ll 1, \\
\left|U_{14}\right|^{2} & \approx \frac{1}{4} \sin ^{2} 2 \theta_{14}
\end{aligned}
$$

Assuming that $m_{4}^{2} \approx \Delta m_{14}^{2}$ we can estimate sterile neutrino mass $m_{4}=(2.68 \pm 0.13) \mathrm{eV}$. In case of parameter $\sin ^{2} 2 \theta_{14} \approx 0.19 \pm$ $0.04(4.6 \sigma)$ obtained combining the results of the Neutrino-4 experiment and results of gallium anomaly measurements and more importantly using value $\Delta m_{14}^{2} \approx(7.2 \pm 0.7) \mathrm{eV}^{2}$ obtained for the first time in the Neutrino-4 experiment, we can make an estimation of the electron neutrino mass: $m_{v_{e}}^{\text {eff }}=(0.58 \pm 0.09) \mathrm{eV}$. Obtained neutrino mass does not contradict the restriction on neutrino mass $m_{v_{e}}^{\text {eff }} \leq 1.1 \mathrm{eV}(C L 90 \%)$ obtained in the KATRIN experiment [16]. Moreover, the results of the determination of the sterile neutrino parameters make it possible to predict the value that can be obtained in the KATRIN experiment.

\section{ACKNOWLEDGEMENTS}

The authors are grateful to the Russian Foundation of Basic Research for support under Contract No. 14-22-03055-ofi_m. Authors are grateful to M.V. Danilov, Y.G.Kudenko, V.B.Brudanin, V.G.Egorov, Y.Kamyshkov, V.A.Shegelsky, V.V. Sinev and D.S. Gorbunov for beneficial discussion of experimental results. The delivery of the scintillator from the laboratory headed by Prof. Jun Cao (Institute of High Energy Physics, Beijing, China) has made a considerable contribution to this research.

\section{References}

[1] I. Alekseev et. al.,Phys. Lett.B 787, 56 (2018).

[2] Y. J. Ko et al., Phys. Rev. Lett 118, 121802 (2017).

[3] J. Ashenfelter et. al., Phys. Rev. Lett. 121, 251802 (2018).

[4] H. Almazán et. al., arXiv:1912.06582.

[5] Y. Abreu et. al., arXiv:2002.05914. 
[6] V. Barinov et al., Phys. Rev. D 99, 111702(R) (2019).

[7] A. P. Serebrov, et. al., arXiv:1708.00421.

[8] A. Serebrov et. al., JETP Lett 109, 213 (2019).

[9] W. Hampel et al., Phys.Lett. B 420, 114-126 (1998).

[10] J. Abdurashitov et al., Phys.Rev. C 59, 2246-2263 (1999).

[11] T. Mueller, D. Lhuillier, M. Fallot et al., Phys. Rev. C 83, 054615 (2011).

[12] G. Mention, M. Fehner, Th. Lasserre et al., Phys. Rev. D 83, 073006 (2011).

[13] S. Gariazzo, C. Giunti, M. Laveder and Y.F. Lie, JHEP 135 (2017).

[14] Particle Data Group, Chapter 14. M. Tanabashi et. al., Phys. Rev. D 98, (030001) (2018) and 2019 update, page $42-47$.

[15] S.Haystotz et al., arXiv:2003.02289.

[16] M. Aker et al., Phys. Rev. Lett. 123, 221802 (2019). 